\title{
Selling to Consumers with Endogenous Types*
}

\author{
Jan Boone ${ }^{\dagger}$ and Joel Shapiro ${ }^{\ddagger}$
}

August 1, 2006

\begin{abstract}
For many goods (such as experience goods or addictive goods), consumers' preferences may change over time. In this paper, we examine a monopolist's optimal pricing schedule when current consumption can affect a consumer's valuation in the future and valuations are unobservable. We assume that consumers are anonymous, i.e. the monopolist can't observe a consumer's past consumption history. For myopic consumers, the optimal consumption schedule is distorted upwards, involving substantial discounts for low valuation types. This pushes low types into higher valuations, from which rents can be extracted. For forward looking consumers, there may be a further upward distortion of consumption due to a reversal of the adverse selection effect; low valuation consumers now have a strong interest in consumption in order to increase their valuations. Firms will find it profitable to educate consumers and encourage forward looking behavior.
\end{abstract} tion

Keywords: endogenous types, experience goods, addictive goods, price discrimina-

J.E.L. codes: D42, D82, L12

${ }^{*}$ We thank Simon Board, Eric Danan, Doh-Shin Jeon, Jens Josephson, Thijs Van Rens, and audiences at Groningen, IIOC, and UPF for helpful comments. Boone gratefully acknowledges financial support from the Dutch National Science Foundation (Grant numbers 016.025.024 and 453.03.606). Shapiro gratefully acknowledges financial support from DGES and FEDER under BEC2003-00412 and Barcelona Economics (CREA).

${ }^{\dagger}$ CentER, TILEC, Tilburg University, ENCORE, UvA, IZA and CEPR. Email: j.boone@uvt.nl

${ }^{\ddagger}$ Departament d’Economia i Empresa, Universitat Pompeu Fabra and CEPR. E-mail: joel.shapiro@upf.edu 


\section{Introduction}

For many goods, consumers' preferences may change over time. These changes can be related to learning how much one needs of the good or some property of the good that gives one an inclination to purchase more. In the first case, we might imagine most types of experience goods (such as electronics or new types of cuisines), in which one experiments with lower quantities or qualities at first to gauge one's interest. In the second case, we might think of addictive goods (such as exercise or caffeine) where previous consumption increases one's desire for subsequent consumption. In this paper we analyze monopoly pricing under asymmetric information when consumers' current consumption influences their future enjoyment of the good.

There are two critical features to our analysis:

- Types are determined by past actions: more consumption today gives a larger probability of a higher valuation for the good in the next period. This is an intensive margin that presents a trade-off for the monopolist - with heterogeneous consumers and asymmetric information about their valuations, downwards distortion of consumption usually solves the adverse selection problem, but may now hurt the firm's future profits.

- Consumers are anonymous: this implies that the monopolist can't make current prices contingent on the consumption history of a customer. One might think of the monopolist as engaging in repeated relationships that remain anonymous, perhaps because the firm distributes through different retailers or posts prices that are available to all consumers (as in the case electronics or restaurants). The monopolist must then choose prices that balance getting low valuation consumers 'hooked' while extracting surplus from high valuation consumers.

Using these elements we find the monopolist's optimal steady-state pricing schedule. We look at both the case where consumers are myopic (they only consider today's utility), and where they are perfectly forward looking. When types are observable, the monopolist increases quality substantially (compared to the static first best get consumers 'addicted'. We find that in the case of myopic consumers, the adverse selection problem may still distort the quality schedule downwards, but not as much as in the static case. In order to entice low valuation customers to begin upgrading, the monopolist may offer substantial discounts, which we define as pricing below cost. In the case of forward looking consumers, we find a remarkable result: the adverse selection problem distorts the quality schedule upwards (compared to static first best outcome). This distortion is driven by the fact that low valuation forward looking consumers have a strong preference to consume and realize type upgrades. In addition we show that it is profitable for the monopolist to educate consumers and make them forward looking. Note, of course, that we assume that there is no harmful aspect to increased consumption in the paper.

Examples of products for which current consumption affects future consumption abound. In software, users often begin using a stripped down version of a program (offered at a substantial

\footnotetext{
${ }^{1}$ The static first best is defined here as marginal (direct) utility equal to marginal costs for each type.
} 
discount) before moving on the full version.] Websites offer basic access (usually for free) with advertisements for those only browsing, but offer a chance to pay for premium content and reduced advertising. The electronics market consists of many products that have both reduced functionality and full functionality versions. Higher end car manufacturers often have a low cost entry level car to attract customers to the brand and persuade them to "trade up" in the future. Similarly, people connecting to the internet often start with a slow connection and then upgrade over time as they start using the internet more and more intensely. And of course, purely addictive substances such as caffeine, exercise or cigarettes have these properties.

Naturally, we must add a caveat to our examples. Previous authors have noted price discrimination in these markets and provided different explanations. The majority of the explanations rely on taste heterogeneity. What we provide is another, complementary explanation and a general framework in which to think about pricing goods whose consumption affects preferences.

In the dynamic mechanism design literature, papers generally either address agents whose types remain the same over time or agents whose type is redrawn from a random distribution in each period. When types are constant over time, short term contracts are influenced by the "ratchet effect": agents' consumption becomes a signal of their type. Freixas, Guesnerie, and Tirole (1985), Laffont and Tirole (1988), and Skreta (2005) analyze this problem. We avoid the signaling problem by assuming that the firm can't make its mechanism dependent on previous consumption. This allows us to focus on the interaction between consumption and type and makes the analysis more tractable. Townsend (1982) models dynamic contracts where agents' types are generated by a random process. His work was extended by Green (1987), Thomas and Worrall (1990), and Atkeson and Lucas (1992). The recent literature on dynamic optimal taxation also assumes that individuals' types are randomly re-drawn each period. A review of this literature is provided by Kocherlakota (2005).

There are a couple of models that incorporate a learning by doing effect on types that is similar to our consumption effect. Baron and Besanko (1984) describe a two period model of regulation where the firm's choice of $R \& D$ in period 1 can affect its type in period 2. This differs from our analysis in two ways. First, the firms' choice of R\&D doesn't interact with the firm's first period type; in our model it is important that current consumption interacts with the consumer's valuation of the good. Second, they analyze the case where the regulator commits to the two period mechanism (to avoid the ratchet effect) but allows the instruments in period 2 to depend on reports of type from both period 1 and period 2. Lewis and Yildirim

\footnotetext{
${ }^{2}$ Intuit's program Quicken was offered as Basic Quicken (\$20) and Quicken Deluxe $(\$ 60)$. Wolfram Research sells both Mathematica and a student version of it (which has a lesser computational speed and reduced functionality). For more examples, see Shapiro and Varian (1998).

${ }^{3}$ For example www.newyorktimes.com, www.salon.com, and www.thestreet.com are three newspaper websites which offer different levels of access at different prices.

${ }^{4}$ This includes the phenomena of damaged goods, in which the firm manufactures the fully functional product and then creates an additional product by adding something to impair the functionality of the original. The classic cases are those of Intel's 486SX chip and IBM's Laserprinter E. See Deneckere and McAfee (1996) for more details.

${ }^{5}$ Keller (2003) cites the case of BMW and its 3-series automobiles, as well as Mercedes and its A-Class vehicles.
} 
(2002) analyze a dynamic model where there is renegotiation each period, types are redrawn randomly each period, and the learning by doing reduction of costs is random and doesn't interact with the firm's type.

Lastly, our model has some similarities to the literature on rational addiction initiated by Becker and Murphy (1988). In our model, current consumption increases future consumption (in expectation), so the goods we discuss have the property of addiction. We look at "beneficial" addictive goods in the sense that utility is increasing in the stock of previous consumption. Our focus, nevertheless is quite different from Becker and Murphy (1988). We focus on monopoly price discrimination, while they assume consumers are homogeneous and prices are competitive. In addition, we look at the steady state, while they are also interested in out of steady state behavior. There are several follow up papers to Becker and Murphy (1988) which look at pricing, but only in the case of homogeneous consumers. These include Becker, Grossman, and Murphy (1994), Fethke and Jagannathan (1996), Showalter (1999), and Driskill and McCafferty (2001).

The paper is organized as follows: Section 2 sets up the model and finds the steady state distribution of types. Section 3 looks at the optimal quality schedule when consumers are myopic. Section 4 examines the solution when consumers are forward looking. Section 5 concludes.

\section{The Model}

Consider a monopolist that sells a product to consumers with utility function $u(x, n)=n x-p$ where $x \geq 0$ is the quality of the good, $n \in\left[n_{0}, n_{1}\right]$ is the valuation of the consumer for the product and $p$ is the price paid for the quality $x$. 'f The monopolist's cost function is represented by $c(x)$, which is assumed to be increasing and convex. We assume that the outside option of all consumers is equal to zero.

The good has addictive properties in the sense that the amount $x$ consumed today affects the consumer's type $n$ tomorrow. The higher today's consumption, the higher the consumer's expected type tomorrow. Hence, for the firm, the density (distribution) function $f(n)(F(n)$ ) over types $\left[n_{0}, n_{1}\right]$ is endogenous. By changing the quality vector $x($.$) , the firm changes the$ distribution of types.

In particular, we assume that consumers live in continuous time $\tau$ and at each instant they have a poisson arrival rate $\delta d \tau$ of a taste shock that can reset their type. For each type $n$ that isn't affected by the taste shock, there is a probability $\phi(x(n))$ that its type increases (a type upgrade) by $d n=\alpha(n) d \tau$ over the next instant. We assume that $\phi^{\prime}(x) \geq 0$, i.e. consuming more quality leads to a higher probability of increasing one's type. This is the learning-byconsuming or addiction effect that we described previously. We also assume that low $n$ types can experience bigger upgrades than high $n$ types, i.e. $\alpha^{\prime}(n) \leq 0$. Furthermore, low types cannot overtake higher types that also experience an upgrade, i.e. $\quad d(n+\alpha(n) d \tau) / d n=1+\alpha^{\prime}(n) d \tau \geq 0$.

\footnotetext{
${ }^{6}$ Alternatively, we could interpret $x$ as the quantity purchased of the good (with $n$ being an individual's valuation per unit purchased).
} 
Finally, we assume that there is a finite upper bound $n_{1}$ on the taste parameter. This implies that $\alpha\left(n_{1}\right)=0$ and no type can end up above $n_{1}$.

In the case where a taste shock does occur, the consumer switches from his current type $n$ to a newly drawn type from an exogenously given distribution $G($.$) over \left[n_{0}, n_{1}\right]$ and corresponding density function $g($.$) . In general, this exogenous distribution may have positive density only on$ a subset of types $\left[n_{0}, \varepsilon\right]$, where $\varepsilon \leq n_{1}$. Below we sometimes focus on the case where $\varepsilon$ is close to $n_{0}$. In that case, the shock $\delta$ leads to a reduction in $n$ for most types.

We assume that the price-quality schedule doesn't depend on a consumer's consumption history. In addition, we assume that the firm is sufficiently patient and maximizes with respect to the steady state distribution. These assumptions are made for tractability reasons. Given that types are not randomly drawn each period, we would like to avoid the well known "ratchet effect". The complexity of a dynamic optimization problem over types and time also disappears with this formulation. Nevertheless, we also have a compelling motivation for not allowing the mechanism to be time dependent. The inability to identify past consumers and their purchasing patterns is a large concern for retailers. Internet retailers have an easier time identifying consumers' purchases, but attempts to condition prices on consumption history have brought substantial controversy?.

To derive the steady state distribution, we first consider how the distribution of types $F($. varies over time. The following equation shows this time variation over a short instant $d \tau$. To derive this, it is convenient to temporarily write the distribution as a function of both type $n$ and time $\tau$. The amount of consumers below type $n$ at time $\tau+d \tau$ is given by

$$
F(n, \tau+d \tau)=\delta d \tau G(n)+(1-\delta d \tau)\left(F(n, \tau)-\int_{n-\alpha\left(n^{\prime}\right) d \tau}^{n} \phi(x(\nu)) f(\nu, \tau) d \nu\right)
$$

There is a probability $\delta d \tau$ that a type $n$ gets a taste shock and is redrawn from $G($.$) . Hence$ each instant there are $\delta d \tau G(n)$ 'new' types below $n$. Of the part that is not affected by this taste shock, only the group who had types larger than $n-\alpha\left(n^{\prime}\right) d \tau$ (where $n^{\prime}$ is defined by $\left.n^{\prime}+\alpha\left(n^{\prime}\right) d \tau=n\right)$ find that a type upgrade $\alpha\left(n^{\prime}\right) d \tau$ is big enough to move beyond $n$. A fraction $\phi(x(\nu))$ of these types experience such an upgrade and move above $n$. The integral subtracts exactly this group. Hence at $\tau+d \tau$ there are two types of consumers who used to be (at time $\tau$ ) below $n$ and are now above it - the group who experiences a taste shock and draws a type larger than $n$ from $G($.$) , and the types \nu \in\left\langle n-\alpha\left(n^{\prime}\right) d \tau, n\right]$ who get a type upgrade. The equation above can be rewritten as:

$$
\begin{aligned}
\frac{F(n, \tau+d \tau)-F(n, \tau)}{d \tau}= & \delta(G(n)-F(n))-\frac{1}{d \tau} \int_{n-\alpha\left(n^{\prime}\right) d \tau}^{n} \phi(x(\nu)) f(\nu, \tau) d \nu+ \\
& \delta \int_{n-\alpha\left(n^{\prime}\right) d \tau}^{n} \phi(x(\nu)) f(\nu, \tau) d \nu
\end{aligned}
$$

\footnotetext{
${ }^{7}$ The most famous example is Amazon.com's attempt at dynamic pricing. When consumers and consumer groups learned that the same good was being offered at different prices depending on consumption history at Amazon, complaints forced Amazon to rescind the policy and offer rebates to those who had previously been affected (see "Amazon backs off on price-testing efforts", by Deborah Kong, USA Today, September 29, 2000).
} 
We take the limit $d \tau \rightarrow 0$ and find (note that the third term on the right hand side converges to zero as $d \tau \rightarrow 0)$ :

$$
\frac{d F(n, \tau)}{d \tau}=\delta(G(n)-F(n))-\phi(x(n)) f(n) \alpha(n)
$$

The steady state distribution is constant over time, i.e. $\frac{d F(n, \tau)}{d \tau}=0$. Thus we find that for each $n$ it must be the case that:

$$
\delta(G(n)-F(n))=\alpha(n) \phi(x(n)) f(n)
$$

This equation says that in the steady state the net inflow of people to types below $n$ due to a taste shock equals the outflow of types due to the upgrading effect.

\section{Myopic Consumers}

\subsection{The Static Benchmarks}

In this section we provide two benchmarks. In both, we consider a simplified model, where both the firm and the consumer are myopic. We define myopic as only being concerned about current period (instant) returns. Therefore the firm takes the distribution of types $F($.$) as$ given. That is, it does not understand the relation between consumption and types given by the distribution in equation (11). The myopic consumer not only doesn't take into account future utility, he doesn't link current consumption to future happiness.

The firm maximizes:

$$
\max _{x(.), p(.)} \int_{n_{0}}^{n_{1}}[p(n)-c(x(n))] f(n) d n
$$

Taking into account that $u(n)=n x(n)-p(n)$, the objective function of the firm becomes:

$$
\max _{x(.), u(.)} \int_{n_{0}}^{n_{1}}[-u(n)+n x(n)-c(x(n))] f(n) d n
$$

In the static first best (SFB) framework, the firm can observe individuals' types. The solution is straightforward; the firm sets every agent's utility equal to zero (making the participation constraint bind) and sets the quality such that $n-c^{\prime}\left(x^{S F B}(n)\right)=0$. The quality $x^{S F B}(n)$ is increasing in $n$, as are prices.

In the static second best (SSB) framework, the firm can't observe the types of consumers. Thus, the monopolist is subject to the incentive compatibility (IC) constraint that each type $n$ chooses the right $(x(n), p(n))$ combination from the menu $(x(),. p()$.$) offered. \mathrm{A}$ A consumer of type $n$ announces type $\hat{n}$ to maximize utility $u(n)$ given by

$$
u(n)=\max _{\hat{n}}[n x(\hat{n})-p(\hat{n})]
$$

\footnotetext{
${ }^{8}$ Using the revelation principle, we can indeed focus on such direct revelation mechanisms.
} 
Hence we find that in an IC mechanism it is the case that

$$
u^{\prime}(n)=x(n)
$$

Since there is no reason to leave surplus to the lowest type $n_{0}$, we can solve for $u(n)$ as

$$
u(n)=\int_{n_{0}}^{n} x(t) d t
$$

We can write the firm's optimization problem as

$$
\max _{x(.)} \int_{n_{0}}^{n_{1}}\left[n x(n)-\int_{n_{0}}^{n} x(t) d t-c(x(n))\right] f(n) d n
$$

which can be rewritten as

$$
\max _{x(.)} \int_{n_{0}}^{n_{1}}\left[\left(n-\frac{1-F(n)}{f(n)}\right) x(n)-c(x(n))\right] f(n) d n
$$

Denoting the solution in this case by $S S B$, we can write

$$
n-c^{\prime}\left(x^{S S B}(n)\right)=\frac{1-F(n)}{f(n)}
$$

provided that $x^{S S B}(n)$ is nondecreasing in $n$ to guarantee that the solution is incentive compatible (see Fudenberg and Tirole (1991: 261)). If $x^{S S B}$ is decreasing in $n$ over some range, the solution needs to be "ironed out" using the procedure described in Fudenberg and Tirole (1991: 303-306). We do not explicitly characterize the solution in this case, but assume throughout that $d x^{S S B}(n) / d n \geq 0$.

Although the firm disregards how the schedule affects the distribution of types, we haven't assumed this effect away. In equation (17), it is clear that the distribution also affects the optimal schedule. We provide an example that solves for the optimal schedule given the distribution effect.

Example 1 Consider the case where $\left[n_{0}, n_{1}\right]=[10,11]$ and $G(n)=1$ for $n \geq 10.1$.9 Further assume that $\alpha(n)=0.1(11-n), \phi(x)=x, \delta=1$ and $c(x)=\frac{1}{2} x^{2}$. Hence we find $x^{S S B}(n)=$ $\frac{n}{1+0.1(11-n)}$ for $n \geq 10.1$. In figure 1 the dotted curve gives this solution. We also plot the static first best solution $x^{S F B}$, which is strictly larger than $x^{S S B}$ except at the top of the valuation distribution.

\footnotetext{
${ }^{9}$ We think of 10.1 as being close to $n_{0}$. In fact, we could have chosen $n_{0}=10.099$ to make it closer to 10.1 and the solution for $n \geq 10.1$ would have been unaffected. The point is that for values of $n$ with $G(n)=1$, the solution $x^{S S B}(n)$ does not depend on the details of the distribution $G($.$) .$
} 


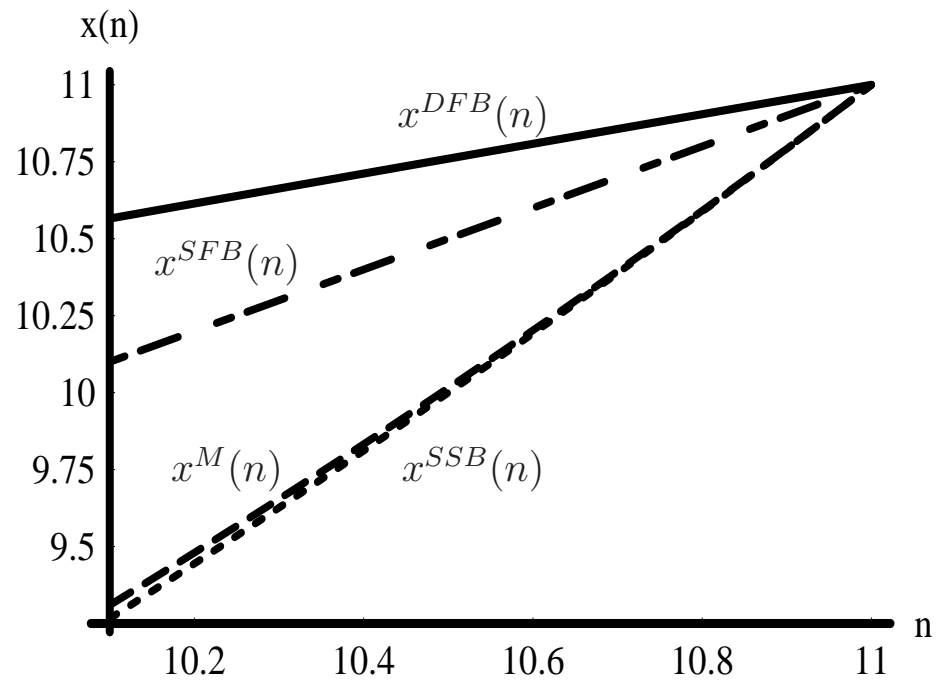

Figure 1: Consumption level $x$ as function of taste parameter $n \geq 10.1$ for the DFB case (solid), the SFB case (dot-dashed), the $M$ case (dash) and the $S S B$ case (dot).

\subsection{First Best with a Forward Looking Firm}

We now allow the firm to maximize long run profits and take into account the type upgrading effect. We assume that the instantaneous discount rate is $\rho \in\langle 0,1\rangle$ and that types are observable. When the firm contracts with a consumer of type $n$, its discounted profits are:

$$
\begin{aligned}
\pi(n)= & (p(n)-c(x(n))) d \tau+(1-(\rho+\delta) d \tau)\{(1-\phi(x(n))) \pi(n)+\phi(x(n)) \pi(n+\alpha(n) d \tau)\} \\
& +\delta d \tau \int_{n_{0}}^{n_{1}} \pi(m) g(m) d m
\end{aligned}
$$

The first term represents the current profits from a consumer of type $n$. The second term is the profits if the consumer doesn't have a taste shock - with probability $\phi(x(n))$ he has a taste upgrade to $n+\alpha(n) d \tau$ and otherwise he remains the same type. The third term is the expected value of profits if the consumer has a taste shock. We note that the probabilities $(1-(\rho+\delta) d \tau)$ and $\delta d \tau$ come from linearizing $e^{-(\rho+\delta) d \tau}$ and $e^{-\rho d \tau}-e^{-(\rho+\delta) d \tau}$, respectively. Manipulating the expression and dividing by $d \tau$ yields:

$$
(\rho+\delta) \pi(n)=p(n)-c(x(n))+\phi(x(n)) \alpha(n) \pi^{\prime}(n)+\delta \int_{n_{0}}^{n_{1}} \pi(m) g(m) d m
$$

where we have used the Taylor approximation $\pi(n+\alpha(n) d \tau)-\pi(n)=\alpha(n) d \tau \pi^{\prime}(n)$ since $d \tau$ is small. Using this expression we can write the discounted total profits by integrating over types:

$$
\begin{aligned}
(\rho+\delta) \int_{n_{0}}^{n_{1}} \pi(n) f(n) d n= & \int_{n_{0}}^{n_{1}}\left\{p(n)-c(x(n))+\phi(x(n)) \alpha(n) \pi^{\prime}(n)\right. \\
& \left.+\delta \int_{n_{0}}^{n_{1}} \pi(m) g(m) d m\right\} f(n) d n
\end{aligned}
$$


We can simplify the expression by using equation (1) for the steady state distribution of types, substituting for $\phi(x(n)) \alpha(n)$ :

$$
\begin{aligned}
(\rho+\delta) \int_{n_{0}}^{n_{1}} \pi(n) f(n) d n= & \int_{n_{0}}^{n_{1}}\{p(n)-c(x(n))\} f(n)+\delta(G(n)-F(n)) \pi^{\prime}(n) d n \\
& +\delta \int_{n_{0}}^{n_{1}} \pi(m) g(m) d m
\end{aligned}
$$

After integrating by parts, we can rewrite in a very straightforward way:

$$
\int_{n_{0}}^{n_{1}} \pi(n) f(n) d n=\frac{1}{\rho} \int_{n_{0}}^{n_{1}}\{p(n)-c(x(n))\} f(n) d n
$$

Therefore the discounted total profits is simply the discounted total revenues minus the discounted total costs. This shouldn't be very surprising, given that in the steady state the distribution of types remains the same at each instant. To eliminate prices, once again we substitute the utility function, making profits equal to:

$$
\frac{1}{\rho} \int_{n_{0}}^{n_{1}}\{n x(n)-u(n)-c(x(n))\} f(n) d n
$$

Since the firm knows the type of each agent, it can make the participation constraint bind, setting $u(n)=0$ for all $n$. This makes the maximization problem (taking into account the effect on the steady state distribution of types):

$\max _{x(.), F(.), f(.)} \int_{n_{0}}^{n_{1}}(n x(n)-c(x(n))) f(n)+\lambda(n)\left(F^{\prime}(n)-f(n)\right)+\mu(n)(\alpha(n) \phi(x(n)) f(n)-\delta(G(n)-F(n))) d n$

This is an optimal control problem. The state variables are $F(n)$ and $x(n)$ and the control variable is $f(n)$. The costate variable for $F(n)$ is $\lambda(n)$, and $\mu(n)$ is the Lagrange multiplier associated with the steady state distribution of types constraint. The endpoints of $F(n)$ are defined: $F\left(n_{0}\right)=0$ and $F\left(n_{1}\right)=1$. The first order conditions (Euler equations) for $x, F$ and $f$ can be written as follows.

$$
\begin{aligned}
n-c^{\prime}(x(n)) & =-\mu(n) \phi^{\prime}(x(n)) \alpha(n) \\
\lambda^{\prime}(n) & =\mu(n) \delta \\
n x(n)-c(x(n))-\lambda(n)+\mu(n) \alpha(n) \phi(x(n)) & =0
\end{aligned}
$$

Instead of setting the marginal profit on type $n, n-c^{\prime}(x(n))$, equal to zero as in the static first best, the firm now takes into account the effect that more consumption of quality has on type upgrading. The second equation gives the differential equation for $\lambda(n)$. Finally, equation (10) equalizes marginal costs and benefits of a small increase in $f(n)$.

The fact that $\alpha\left(n_{1}\right)=0$ yields that $x\left(n_{1}\right)$ coincides with the static first best solution $\left(n_{1}-c^{\prime}\left(x\left(n_{1}\right)\right)=0\right)$. In addition, the condition $n_{1} x\left(n_{1}\right)-c\left(x\left(n_{1}\right)\right)-\lambda\left(n_{1}\right)=0$ holds.

We can prove that the distortion of $x(n)$ is an upwards distortion from the static first best solution. The main step is to prove that $\mu(n) \geq 0$ or equivalently $n x(n)-c(x(n))-\lambda(n) \leq 0$. We denote the solution as $x^{D F B}(n)$, where DFB signifies "dynamic first best". 
Proposition 1 For all $n \in\left[n_{0}, n_{1}\right\rangle, x^{D F B}(n)>x^{S F B}(n)$.

Proof. We begin by proving that $n x(n)-c(x(n))-\lambda(n) \leq 0$ for all $n$. Suppose not, i.e. $n x(n)-c(x(n))-\lambda(n)>0$ for some $n$. Using equation (10), we see from equation (9), $\lambda^{\prime}(n)<0$ and from equation (8), $n-c^{\prime}(x(n))>0$. Therefore the derivative with respect to $n$ of $n x(n)-c(x(n))-\lambda(n), x(n)+\left(n-c^{\prime}(x)\right) \frac{d x}{d n}-\lambda^{\prime}$, is positive. Since, $n_{1} x\left(n_{1}\right)-c\left(x\left(n_{1}\right)\right)-\lambda\left(n_{1}\right)=0$, we find a contradiction (it can't be that $n x(n)-c(x(n))-\lambda(n)$ is positive and increasing for all $n)$.

Now suppose $n x(n)-c(x(n))-\lambda(n)=0$ for any $n \in\left[n_{0}, n_{1}\right\rangle$. This implies that $\mu(n)=0$. Taking the derivative of $n x(n)-c(x(n))-\lambda(n)=0$ yields the equation $n-c^{\prime}(x(n))=\frac{-x(n)}{x^{\prime}(n)}$, using $\mu(n)=0$ and equation (9 ). However, since $\mu(n)=0$, equation (8) gives us $n-c^{\prime}(x(n))=0$. This gives us a contradiction.

Therefore, for all $n \in\left[n_{0}, n_{1}\right\rangle, n x(n)-c(x(n))-\lambda(n)<0$ and $\mu(n)>0$. Using this fact and equation (8) proves the proposition.

This demonstrates that the dynamic first best sells higher qualities to all consumers (except the highest valuation consumer) than the static first best. This is intuitive since the firm now takes into account the fact that raising $x(n)$ makes it more likely that type $n<n_{1}$ upgrades to a higher (and more profitable) type. In addition, the quality schedule is strictly greater than the static second best schedule for all $n$ except for $n_{1}$.

If, in this case, a quality level $\bar{x}$ was reached where any additional quality changes would have no effect in changing the probability of upgrading (i.e. $\phi^{\prime}(x)=0$ for $x>\bar{x}$ and $\bar{x}<x^{D F B}\left(n_{1}\right)$ ), the upward distortion of quality would disappear. That is, if $x(\hat{n})=\bar{x}$, then for all $n>\hat{n}$, qualities would be equal to those of the static first best.

We now look at an example of the schedule we described.

Example 2 Using the same parameter and functional choices as in example 1 we derive

$$
\lambda(n)=\frac{1}{2}(x(n))^{2}
$$

Thus we find

$$
\lambda^{\prime}(n)=x(n) x^{\prime}(n)
$$

Combining this with equation (9) we find the following differential equation for $x(n)$ :

$$
x^{\prime}(n)=\frac{1}{\alpha(n)}\left(1-\frac{n}{x(n)}\right)
$$

with boundary condition $x\left(n_{1}\right)=n_{1}$. The solution of this differential equation, $x^{D F B}(n)$, is the solid line in figure 1 . 


\subsection{Second Best with a Forward Looking Firm}

We now assume that the monopolist is forward looking and knows that consumers are myopic, but doesn't observe their valuations. The monopolist's task is to encourage consumption and make consumers realize these type enhancements in order to extract rents from them while taking into account incentives for consumers to misstate their type. Incentive Compatibility is still given by $u^{\prime}(n)=x(n)$. Hence the optimal control problem for the firm (once again setting $\left.u\left(n_{0}\right)=0\right)$ is as follows.

$$
\begin{aligned}
& \max _{x(.), F(.), f(.)} \int_{n_{0}}^{n_{1}}[n x(n)-c(x(n))] f(n)-(1-F(n)) x(n)+ \\
& \lambda(n)\left(F^{\prime}(n)-f(n)\right)+\mu(n)(\alpha(n) \phi(x(n)) f(n)-\delta(G(n)-F(n)) d n
\end{aligned}
$$

As above, the endpoints of $F(n)$ are defined: $F\left(n_{0}\right)=0$ and $F\left(n_{1}\right)=1$. The first order conditions (Euler equations) for $x, F$ and $f$ can be written as follows.

$$
\begin{aligned}
n-c^{\prime}(x(n)) & =\frac{1-F(n)}{f(n)}-\mu(n) \alpha(n) \phi^{\prime}(x(n)) \\
\lambda^{\prime}(n) & =x(n)+\delta \mu(n) \\
n x(n)-c(x(n))-\lambda(n)+\mu(n) \alpha(n) \phi(x(n)) & =0
\end{aligned}
$$

The first equation equates the margin $n-c^{\prime}(x(n))$ to the informational rent and the type upgrading margin (which we saw in the dynamic first best). The second equation gives the differential equation for $\lambda(n)$. Equation (13) equalizes marginal costs and benefits of a small increase in $f(n)$.

The shape of the optimal quality schedule can be compared easily to our benchmarks. Consider $n=n_{1}$. Since $\alpha\left(n_{1}\right)=0$, it must be that $n_{1}-c^{\prime}\left(x\left(n_{1}\right)\right)=0$. This proves that there is a "no distortion at the top" result, where the quality consumed by the highest type here is equivalent to that consumed by the highest type in the dynamic first best (as well as the static first best and the static second best). We also note that equation (13) implies that $n_{1} x\left(n_{1}\right)-c\left(x\left(n_{1}\right)\right)-\lambda\left(n_{1}\right)=0$. We denote the solution as $x^{M}(n)$, where $M$ stands for myopic. As with the $S S B$ case above, for $x^{M}(n)$ to be incentive compatible, we need $d x^{M}(n) / d n \geq 0$. If the solution generated by the equations above, is decreasing in $n$ over some range, it needs to be "ironed out" to get $x^{M}$. We assume that $d x^{M}(n) / d n \geq 0$ holds for all $n$ and below derive conditions under which $x^{M}$ is strictly increasing in $n$. The following lemma helps us to get some insight into the shape of $x^{M}(n)$.

Lemma 1 For each $n$ it is the case that

$$
n x(n)-c(x(n))-\lambda(n) \leq 0
$$

with strict inequality for some non-empty interval. 
Proof. We first prove the weak inequality holds by contradiction. Suppose not, i.e. suppose there exists $n^{\prime}$ such that $n^{\prime} x\left(n^{\prime}\right)-c\left(x\left(n^{\prime}\right)\right)-\lambda\left(n^{\prime}\right)>0$. Isolate $\mu\left(n^{\prime}\right)<0$ in equation (13) and substitute it into equation (12). This implies that $\lambda^{\prime}\left(n^{\prime}\right)<x\left(n^{\prime}\right)$. Hence we find

$$
\frac{d\left(n^{\prime} x\left(n^{\prime}\right)-c\left(x\left(n^{\prime}\right)\right)-\lambda\left(n^{\prime}\right)\right)}{d n^{\prime}}>\left(n^{\prime}-c^{\prime}\left(x\left(n^{\prime}\right)\right)\right) x^{\prime}\left(n^{\prime}\right) \geq 0
$$

where the last inequality follows from $n^{\prime}-c^{\prime}\left(x\left(n^{\prime}\right)\right) \geq 0$ (see equation (11)) and $x^{\prime}\left(n^{\prime}\right) \geq 0$. However, $n x(n)-c(x(n))-\lambda(n)>0$ and increasing in $n$ contradicts $n_{1} x\left(n_{1}\right)-c\left(x\left(n_{1}\right)\right)-\lambda\left(n_{1}\right)=$ 0 .

Next, we prove that the strict equality holds for some non-empty interval. If it didn't, then $n x(n)-c(x(n))-\lambda(n)=0$ for all $n$. This implies that $\mu(n)=0$ for $n \in\left\langle n_{0}, n_{1}\right\rangle$. Taking the derivative of $n x(n)-c(x(n))-\lambda(n)=0$ yields the equation $\left(n-c^{\prime}(x(n))\right) x^{\prime}(n)=0$, using $\mu(n)=0$ and equation (12). But $\mu(n)=0$ also implies $c^{\prime}\left(x\left(n_{0}\right)\right)<n_{0}$. Together with $x^{\prime}(n)=0$ and $c^{\prime}\left(x\left(n_{1}\right)\right)=n_{1}>n_{0}$ this yields a contradiction.

Using equation (11), this result gives us the following:

Proposition 2 Assume that there exists $\tilde{n}<n_{1}$ such that $G(\tilde{n})=1$. Then for all $n \geq \tilde{n}$ the quality schedule $x^{M}(n) \geq x^{S S B}(n)$. Furthermore, there is a non-empty interval where $x^{M}(n)>x^{S S B}(n)$.

The monopolist pushes up the qualities consumed relative to the static second best in order to take advantage of the boost in demand created for its product from high types. This increase in quality doesn't affect the whole schedule. At the top, the consumer is kept at the static efficient point. There is little reason to distort the consumption upwards at the top since this type is not able to benefit from the upgrading effect.

This intuition allows us to explore the schedule further. Imagine that for large enough consumption $x>\bar{x}$, there was no effect in changing the probability of upgrading, i.e. $\phi^{\prime}(x)=0$. Also suppose that $x^{M}\left(n_{0}\right)<\bar{x}<x^{M}\left(n_{1}\right)$, meaning that for types above some cutoff $\hat{n}$, there is no longer a marginal upgrading effect. Then for all $n>\hat{n}, x^{M}(n)=x^{S S B}(n)$ (this can be seen from equation (11)). That is, when there is no marginal upgrading effect, the upwards distortion on consumption is not optimal for the monopolist.

The advantage of the assumption in the above proposition on the cutoff $\tilde{n}$ (where for all $n \geq \tilde{n}$ we have $G(n)=1$ ) is that it yields a very useful simplification. In particular, for $n \geq \tilde{n}$, the hazard rate $\frac{1-F(n)}{f(n)}$ equals $\frac{\alpha(n) \phi(x(n))}{\delta}$ and we can directly characterize the quality schedule. We use it once again in the following lemma to show that the quality schedule is strictly increasing.

Lemma 2 Assume that there exists $\tilde{n}<n_{1}$ such that $G(\tilde{n})=1$. Then $\phi^{\prime \prime}() \leq$.0 implies that $d x^{M}(n) / d n>0$ for all $n \geq \tilde{n}$. 
Proof Substituting for $\frac{1-F(n)}{f(n)}$ and $\mu(n)$ in equation (11) and using the implicit function theorem we find that

$$
\frac{d x^{M}(n)}{d n}=\frac{1-\frac{\alpha^{\prime} \phi}{\delta}+\frac{\phi^{\prime}}{\phi}\left(\lambda^{\prime}-x\right)}{c^{\prime \prime}+\frac{\alpha \phi^{\prime}}{\delta}-\frac{\phi^{\prime \prime} \phi-\phi^{\prime 2}}{\phi^{2}}(\lambda-n x+c(x))+\frac{\phi^{\prime}}{\phi}\left(n-c^{\prime}(x)\right)}
$$

We prove that this expression is positive. First, note that the numerator is positive as $\alpha^{\prime}($. $0, \phi^{\prime}() \geq$.0 and $\lambda^{\prime}(n)-x(n)=\delta \mu(n)$ which is non-negative by lemma 1. Next, turn to the denominator:

$$
c^{\prime \prime}+\frac{\alpha \phi^{\prime}}{\delta}-\frac{\phi^{\prime \prime} \phi-\phi^{\prime 2}}{\phi^{2}} \mu(n) \alpha(n) \phi(x(n))+\frac{\phi^{\prime}}{\phi}\left(n-c^{\prime}(x)\right)
$$

Re-writing:

$$
c^{\prime \prime}+\frac{\alpha \phi^{\prime}}{\delta}-\phi^{\prime \prime} \mu(n) \alpha(n)+\frac{\phi^{\prime}}{\phi} \mu(n) \alpha(n) \phi^{\prime}(x(n))+\frac{\phi^{\prime}}{\phi}\left(n-c^{\prime}(x)\right)
$$

We can combine terms to get:

$$
c^{\prime \prime}+\frac{\alpha \phi^{\prime}}{\delta}-\phi^{\prime \prime} \mu(n) \alpha(n)+\frac{\phi^{\prime}}{\phi}\left(\frac{\alpha(n) \phi(x(n))}{\delta}\right)
$$

Which is then:

$$
c^{\prime \prime}+\frac{2 \alpha \phi^{\prime}}{\delta}-\phi^{\prime \prime} \mu(n) \alpha(n)>0
$$

which is positive given that $c^{\prime \prime} \geq 0$ and $\phi^{\prime \prime} \leq 0$.

Finally, consider the following example to compare the myopic outcome with the schedules derived before.

Example 3 Using the same specifications and parameter values as in the previous examples, we can write the first order conditions as follows for $n \geq 10.1$ :

$$
\begin{aligned}
& \mu(n)=\frac{x(n)-n+\alpha(n) x(n)}{\alpha(n)} \\
& \lambda(n)=x(n)^{2}\left(\frac{1}{2}+\alpha(n)\right)
\end{aligned}
$$

Differentiating the last equation with respect to $n$ and combining it with equation (19) yields the following differential equation in $x($.$) :$

$$
x^{\prime}(n)=\frac{-\alpha^{\prime}(n) x(n)+2+\frac{1-\frac{n}{x(n)}}{\alpha(n)}}{1+2 \alpha(n)}
$$

together with the boundary condition $x\left(n_{1}\right)=n_{1}$. The solution to this differential equation, $x^{M}(n)$, is given by the dashed line in figure 11 . The figure illustrates that $x^{M}(n) \geq x^{S S B}(n)$, with a strict inequality for an interval. 


\subsection{Discounts and prices below cost}

A natural question arises when the monopolist increases quality for low types to encourage upgrading: what do prices look like? Do we observe the phenomena of discounting? Discounts (or introductory offers) often arise in the literature on experience goodsto; however the main explanation given for the use of discounts is the presence of quality uncertainty by consumers. Low prices encourage experimentation and can boost future demand for the firm. Our explanation, that low prices promote upgrading, encompasses this story and allows for addiction effects as well.

The brief answer to our question is that prices can be very low in our model, and in what follows we describe an example where prices can actually be below cost for low types. The monopolist sells at a loss in order to create higher types to extract rents from.

Assume the function $\phi(x)$ takes the form:

$$
\phi(x)=\left\{\begin{array}{l}
0 \text { if } x<\bar{x} \\
\phi \text { if } x \geq \bar{x}
\end{array}\right.
$$

where $\bar{x}$ is the quality level at which upgrading begins. Hence we assume that a consumer who consumes low quality doesn't get a taste for what the high end good could yield. Therefore, such consumption of low quality does not generate an upgrade. An internet user with a poor connection, for example, may never experience the convenience of downloading music and will not be tempted to upgrade her connection.

Furthermore, let the exogenous distribution $G(n)$ be a uniform distribution on $\left[n_{0}, n_{1 g}\right]$, i.e. $G(n)=\frac{n-n_{0}}{n_{1 g}-n_{0}}$, and the cost function be quadratic, $c(x)=\frac{1}{2} x^{2}$. The following assumption reduces the number of cases that we need to consider below.

$$
n_{1 g} \bar{x}-c(\bar{x})=0
$$

This equation implies $\bar{x}=2 n_{1 g}$.

This equation says that if the firm decides to sell $\bar{x}$ to types $n<n_{1 g}$, it sells at a price below costs. Since the maximum one could charge type $n_{1 g}$ would be $n_{1 g} \bar{x}$ (due to the participation constraint), any price below that would lose the firm money. The firm breaks even by extracting all of type $n_{1 g}$ 's surplus, and therefore would lose money if it encouraged lower types to consume $\bar{x}$ and upgrade. This leaves the firm with two options: sell qualities below $\bar{x}$ to all consumers and have no upgrades, or to sell high quality goods and encourage upgrades. The second option, which by construction involves prices below costs, will be more profitable for certain parameters.

First, we examine the case where the firm sells qualities below $\bar{x}$ and no upgrading occurs. The optimal quality schedule is given by:

$$
n-c^{\prime}(x(n))=\frac{1-G(n)}{g(n)}
$$

\footnotetext{
${ }^{10}$ For example, see Shapiro (1983), Schlee (2001), and Gabszewicz, Pepall, and Thisse (1992). Price discrimination in these models is intertemporal. Our model has both static (i.e. within period) and intertemporal price discrimination.
} 


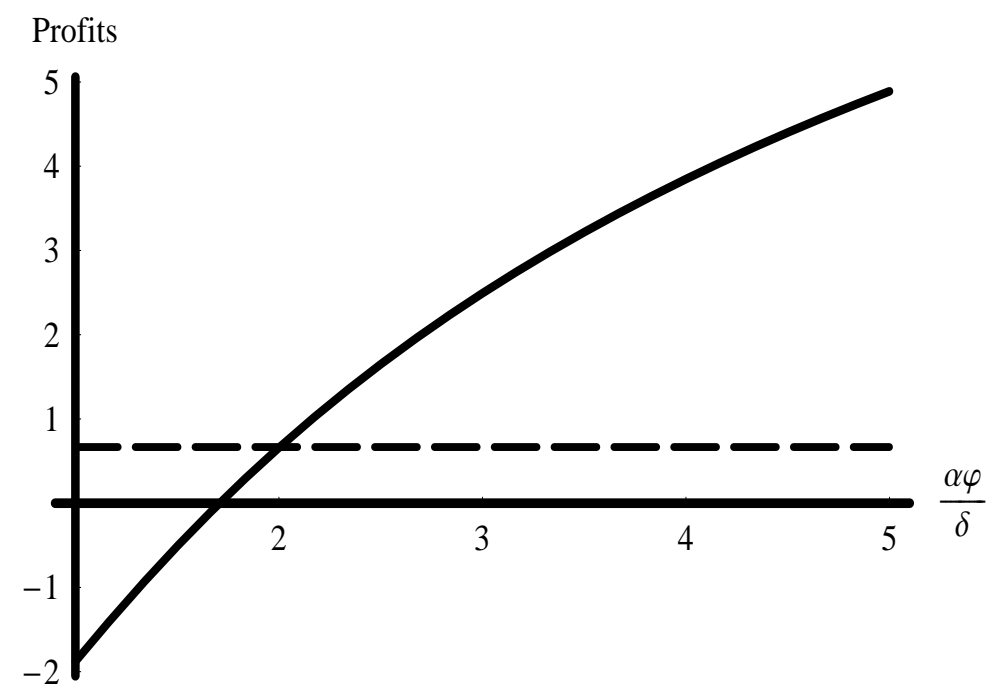

Figure 2: Profits as a function of $\frac{\alpha \phi}{\delta}$ for the case without (dash) and with (solid) upgrading.

Since $x(n)<\bar{x}$ for all $n \in\left[n_{0}, n_{1 g}\right]$, there will not be any upgrading. With the functions that we chose and assuming in addition that $2 n_{0} \geq n_{1 g}$, we find that:

$$
\begin{aligned}
x(n) & =2 n-n_{1 g} \\
p(n) & =n x(n)-\int_{n_{0}}^{n} x(t) d t \\
\text { profit } & =\int_{n_{0}}^{n_{1 g}}[p(n)-c(x(n))] g(n) d n=\left(4 n_{0}^{2}-2 n_{0} n_{1 g}+n_{1 g}^{2}\right) / 6
\end{aligned}
$$

Second, we look at a quality schedule where upgrading occurs. The schedule we choose may not be the optimum, but since we prove that it can yield higher profits than the case without upgrading, the optimum (for those parameters) must involve upgrading. Moreover, since any case with upgrading involves pricing below cost, deep discounts will be a part of the optimal solution (for those parameters).

Suppose all types in the interval $\left[n_{0}, n_{1 g}\right]$ are sold $\bar{x}$. It follows that $p(n)=n_{0} \bar{x}$ for these types, otherwise type $n_{0}$ would not buy. Since $x(n)$ cannot fall (by incentive compatibility) and there is no reason to sell qualities in excess of $\bar{x}$, we find $x(n)=\bar{x}$ for all $n \in\left[n_{0}, \bar{n}\right]$ where $\bar{n}$ is defined by

$$
\bar{n}-c^{\prime}(\bar{x})=\frac{1-F(\bar{n})}{f(\bar{n})}
$$

Thus, the quality schedule for types in the interval $\left[\bar{n}, n_{1}\right]$ is given by $n-c^{\prime}(x)=\frac{1-F(n)}{f(n)}$. To determine $F(n)$ we assume that $\alpha(n)=\alpha \cdot\left(n_{1}-n\right)$ for some scalar $\alpha>0$. Substituting this 
into our steady state equation, we find

$$
F^{\prime}(n)=\frac{\delta}{\phi \alpha\left(n_{1}-n\right)}(G(n)-F(n))
$$

For $n>n_{1 g}, G(n)=1$. Using $F\left(n_{1}\right)=1$ we find that

$$
F(n)=1-C\left(\frac{\alpha \phi}{\delta}\left(n_{1}-n\right)\right)^{\frac{\delta}{\alpha \phi}} \text { for } n>n_{1 g}
$$

for some constant $C>0$ to be determined below.

For $n<n_{1 g}, G(n)=\frac{n-n_{0}}{n_{1 g}-n_{0}}$, yielding

$$
F(n)=\frac{\frac{\alpha \phi}{\delta}\left(n_{1}-n_{0}\right)-\left(n-n_{0}\right)+\frac{\alpha \phi}{\delta}\left(n_{1}-n\right)^{\frac{\delta}{\alpha \phi}}\left(n_{1}-n_{0}\right)^{1-\frac{\delta}{\alpha \phi}}}{\left(\frac{\alpha \phi}{\delta}-1\right)\left(n_{1 g}-n_{0}\right)} \text { for } n \leq n_{1 g}
$$

The constant $C$ is found by the continuity of $F($.$) at n=n_{1 g}$. It turns out that

$$
C=\frac{\left(\frac{\alpha \phi}{\delta}\right)^{1-\frac{\delta}{\alpha \phi}}}{\frac{\alpha \phi}{\delta}-1} \frac{\left(n_{1}-n_{0}\right)^{1-\frac{\delta}{\alpha \phi}}-\left(n_{1}-n_{1 g}\right)^{1-\frac{\delta}{\alpha \phi}}}{n_{1 g}-n_{0}}
$$

Since $\bar{x}=2 n_{1 g}$ we can now solve for $\bar{n}>n_{1 g}$. We know that for $n>n_{1 g}$ it is the case that $\frac{1-F(n)}{f(n)}=\frac{\alpha \phi}{\delta}\left(n_{1}-n\right)$. Thus we find

$$
\bar{n}=\frac{\frac{\alpha \phi}{\delta} n_{1}+2 n_{1 g}}{1+\frac{\alpha \phi}{\delta}}
$$

Hence the monopolist sells the quality $\bar{x}$ at a (total) price $n_{0} \bar{x}$ to a fraction $F\left(\frac{\frac{\alpha \phi}{\delta} n_{1}+2 n_{1 g}}{1+\frac{\alpha \phi}{\delta}}\right)$ of the consumers. Since $n_{0} \bar{x}-c(\bar{x})<0$ the firm makes a loss on these consumers. To types $n>\bar{n}$ the firm sells

$$
x(n)=\left(1+\frac{\alpha \phi}{\delta}\right) n-\frac{\alpha \phi}{\delta} n_{1}
$$

From this we can calculate the profits for $n>\bar{n}$ in the standard way.

Now we can analyze whether it is optimal for the firm to sell $\bar{x}$ to the low types at a loss such that the upgrading can take effect. We consider the parameters $n_{0}=1, n_{1 g}=2$, and $n_{1}=10$. Profits in the no-upgrading solution (when $x(n)<\bar{x}$ for all $n$ ) are then equal to $2 / 3$. Selling $\bar{x}$ to low types leads to profits as depicted in figure 2 . Clearly for $\alpha \phi / \delta$ high enough, it is optimal for the firm to sell to low types at a loss. This is intuitive: the stronger the upgrading effect (because $\alpha \phi / \delta$ is high) the more profitable the strategy that induces upgrading. 


\section{Forward Looking Consumers}

\subsection{First Best}

In this section, we assume that consumers understand perfectly the type upgrading process and take it into account when making consumption decisions. To characterize incentive compatibility in this case, we first derive the expected discounted value of a type $n$ consumer. Denoting this value by $V(n)$, we can write

$$
\begin{aligned}
V(n)= & (n x(n)-p(n)) d \tau+(1-(\rho+\delta) d \tau)((1-\phi(x(n))) V(n)+ \\
& \phi(x(n)) V(n+\alpha(n) d \tau))+\delta d \tau \int_{n_{0}}^{n_{1}} V(m) g(m) d m
\end{aligned}
$$

Hence the expected discounted value of being of type $n$ consists of three parts. First, the consumer receives the instant utility $n x(n)-p(n)$ over the short 'period' $d \tau$. Second, with some probability the consumer experiences no taste shock and gets a type upgrade of $\alpha(n) d \tau$ with probability $\phi(x(n))$, or continues with payoff $V(n)$ with probability $1-\phi(x(n))$. Third, if the consumer experiences the taste shock, the payoff is the expectation of the continuation value $V(m)$, where $m$ is distributed according to the exogenously given density function $g(m)$. Using the standard techniques of deriving a Bellman equation we get the following equation for $V(n)$ :

$$
(\rho+\delta) V(n)=n x(n)-p(n)+\alpha(n) \phi(x(n)) V^{\prime}(n)+\delta \int_{n_{0}}^{n_{1}} V(m) g(m) d m
$$

Rewriting to isolate the price $p(n)$ :

$$
p(n)=n x(n)-(\rho+\delta) V(n)+\alpha(n) \phi(x(n)) V^{\prime}(n)+\delta \int_{n_{0}}^{n_{1}} V(m) g(m) d m
$$

We will use this to eliminate prices from the objective function of the firm. Integrating over types (and using equation (1) and integration by parts) yields:

$$
\int_{n_{0}}^{n_{1}} p(n) f(n) d n=\int_{n_{0}}^{n_{1}}(n x(n)-\rho V(n)) f(n) d n
$$

Expected discounted profits for the firm can then be written as:

$$
\int_{n_{0}}^{n_{1}} \pi(n) f(n) d n=\frac{1}{\rho} \int_{n_{0}}^{n_{1}}(n x(n)-c(x(n))-\rho V(n)) f(n) d n
$$

In the first best solution, the firm maximizes profits subject to the equation representing the steady state distribution of types and the participation constraint. Since types are observable, the firm can set $V(n)=0$ for all $n$. This then makes the problem equivalent to the dynamic first best problem when agents are myopic. The solution therefore is the dynamic first best quality schedule, $x^{D F B}(n)$. 


\subsection{Second Best}

To derive incentive compatibility we take one step back and write:

$$
(\rho+\delta) V(n)=\max _{\tilde{n}}\left\{n x(\tilde{n})-p(\tilde{n})+\alpha(n) \phi(x(\tilde{n})) V^{\prime}(n)+\delta \int_{n_{0}}^{n_{1}} V(m) g(m) d m\right\}
$$

Using the envelope theorem we find the incentive compatibility condition:

$$
(\rho+\delta) V^{\prime}(n)=x(n)+\alpha^{\prime}(n) \phi(x(n)) V^{\prime}(n)+\alpha(n) \phi(x(n)) V^{\prime \prime}(n)
$$

It is straightforward to see that the solution to the first best problem doesn't hold here. Suppose $V(n)=0$ for the whole interval $\left[n_{0}, n_{1}\right]$. This violates the incentive compatibility constraint since it produces the equation $0=x(n)$ for all $n$. Since the monopolist could make positive profits by selling small amounts of the good, it can't be that $x(n)=0$ everywhere.

The following result gives us insight into the value function.

Proposition 3 For functions $V($.$) that are twice differentiable we find that V^{\prime}(n) \geq 0$.

Proof Consider two types $n^{\prime}>n$ where $n^{\prime}$ can mimic $n$. We see that

$$
\begin{aligned}
& (\rho+\delta) V\left(n^{\prime}\right) \geq n^{\prime} x(n)-p(n)+\alpha\left(n^{\prime}\right) \phi(x(n)) V^{\prime}\left(n^{\prime}\right)+\delta \int_{n_{0}}^{n_{1}} V(m) g(m) d m \\
& (\rho+\delta) V(n)=n x(n)-p(n)+\alpha(n) \phi(x(n)) V^{\prime}(n)+\delta \int_{n_{0}}^{n_{1}} V(m) g(m) d m
\end{aligned}
$$

Subtract these two equations and consider the case where $n^{\prime}=n_{1}$ (and thus $\alpha\left(n_{1}\right)=0$ ):

$$
(\rho+\delta)\left(V\left(n_{1}\right)-V(n)\right) \geq\left(n_{1}-n\right) x(n)-\phi(x(n)) V^{\prime}(n) \alpha(n)
$$

If there exists $n$ such that $V^{\prime}(n)<0$ then this equation implies that $V\left(n_{1}\right)>V(n)$. Hence there must exist $n^{\prime \prime} \in\left\langle n, n_{1}\right\rangle$ such that $V^{\prime}\left(n^{\prime \prime}\right)>0$. Since $V^{\prime}(n)<0$ and $V^{\prime}\left(n^{\prime \prime}\right)>0$ it must be the case that over some range $V^{\prime \prime}()>$.0 . In particular, there must exist $\tilde{n}$ such that $V^{\prime}(\tilde{n})<0$ and $V^{\prime \prime}(\tilde{n})>0$. However, this contradicts equation (18). Hence $V^{\prime}(n) \geq 0$ for all $n \in\left[n_{0}, n_{1}\right]$. Q.E.D.

Substituting the fact that $V(n)=V\left(n_{0}\right)+\int_{n_{0}}^{n} V^{\prime}(n) d n$ into the firm's objective function given by equation (16) and integrating by parts gives the firm's maximization problem:

$$
\begin{aligned}
& \max _{x(.), F(.), f(.), \nu(.), \omega(.)}-V\left(n_{0}\right)+\int_{n_{0}}^{n_{1}}[n x(n)-c(x(n))] f(n)-\rho(1-F(n)) \nu(n)+ \\
& \lambda(n)\left(F^{\prime}(n)-f(n)\right)+\mu(n)\left(\alpha(n) \phi(x(n)) f(n)-\delta(G(n)-F(n))+\psi(n)\left(\nu^{\prime}(n)-\omega(n)\right)\right. \\
& +\xi(n)\left[\left(\rho+\delta-\alpha^{\prime}(n) \phi(x(n)) \nu(n)-x(n)-\alpha(n) \phi(x(n)) \omega(n)\right] d n\right.
\end{aligned}
$$


where $\nu(n)=V^{\prime}(n), \omega(n)=\nu^{\prime}(n)=V^{\prime \prime}(n), \psi($.$) is the costate variable associated with$ $\nu(n)$, and $\xi($.$) is a Lagrange multiplier. The firm is constrained by the steady state distribution$ of types equation and incentive compatibility. It is clear from the problem itself that the value function for the lowest type will be set equal to zero (the participation constraint binds and $\left.V\left(n_{0}\right)=0\right)$. In this case, we have five optimality conditions:

$$
\begin{aligned}
\left(n-c^{\prime}(x(n))\right) f(n) & =-\mu(n) \alpha(n) \phi^{\prime}(x(n)) f(n)+\xi(n)\left(1+\alpha^{\prime}(n) \phi^{\prime}(x(n)) \nu(n)+\alpha(n) \phi^{\prime}(x(n)) \omega(n)\right)(19) \\
\lambda^{\prime}(n) & =\rho \nu(n)+\mu(n) \delta \\
0 & =n x(n)-c(x(n))-\lambda(n)+\mu(n) \alpha(n) \phi(x(n)) \\
\psi^{\prime}(n) & =-\rho(1-F(n))+\xi(n)\left(\rho+\delta-\alpha^{\prime}(n) \phi(x(n))\right) \\
0 & =\psi(n)+\xi(n) \alpha(n) \phi(x(n))
\end{aligned}
$$

We elaborate on the solution in the appendix, but go over the important aspects here. Solving the fifth equation for $\xi(n)=-\psi(n) /(\alpha(n) \phi(x(n)))$ and plugging that into the fourth equation gives us a differential equation for $\psi(n)$. The solution to the differential equation takes the following form (using the transversality condition that $\psi\left(n_{1}\right)=0$ ).

$$
\psi(n)=\left(\int_{n}^{n_{1}} \rho(1-F(s)) e^{\int_{n_{0}}^{s} \frac{\rho+\delta-\alpha^{\prime} \phi(x)}{\alpha \phi(x)} d u} d s\right)\left(e^{-\int_{n_{0}}^{n} \frac{\rho+\delta-\alpha^{\prime} \phi(x)}{\alpha \phi(x)} d s}\right)
$$

Therefore, $\psi(n)$ is clearly nonnegative, and consequently $\xi(n)$ must be non-positive. In the following proposition we prove that the term related to incentive compatibility in equation (19),

$$
\frac{d}{d n}\left(-\frac{d V / d x}{d V / d p}\right)=\frac{d\left(n+\alpha(n) \phi^{\prime}(x) V^{\prime}(n)\right)}{d n}=1+\alpha^{\prime}(n) \phi^{\prime}(x(n)) \nu(n)+\alpha(n) \phi^{\prime}(x(n)) \omega(n)
$$

is positive.

Proposition 4 Assume that $\phi^{\prime \prime}() \leq$.0 . Then

$$
1+\alpha^{\prime}(n) \phi^{\prime}(x(n)) V^{\prime}(n)+\alpha(n) \phi^{\prime}(x(n)) V^{\prime \prime}(n) \geq 0 .
$$

\section{Proof}

Substituting for $V^{\prime \prime}(n)$ from equation (18), we write this inequality as

$$
1-\frac{\phi^{\prime}(x) x}{\phi(x)}+V^{\prime}(n)(\rho+\delta) \frac{\phi^{\prime}(x)}{\phi(x)} \geq 0
$$

This equation holds, since $V^{\prime}(n) \geq 0$ and $\phi(x) \geq \phi^{\prime}(x) x$. To see the last inequality we use the following Taylor series

$$
\phi(0)=\phi(x)+\phi^{\prime}(x)(0-x)+\frac{1}{2} \phi^{\prime \prime}(\zeta)(0-x)^{2}
$$


for some $\zeta \in[0, x]$. This can be rewritten as

$$
\phi(x)=\phi(0)+\phi^{\prime}(x) x-\phi^{\prime \prime}(\zeta) x^{2} \geq \phi^{\prime}(x) x
$$

Looking at the first necessary condition (19), using Proposition 1 and $\xi(n) \leq 0$, we can then conclude that the contribution of incentive compatibility is to increase the quality schedule, rather than reduce it (as it does in the myopic case and the static second best).

This reversal is explained by the difference between myopic and forward looking consumers. To dissuade high types from masquerading as low types in the case with myopic consumers (and in the static case as well), the quality schedule is generally distorted downwards, since high types have a strong preference for quality over low types. Here however, low types have a strong preference for quality as well, since they benefit both from the quality now and the type upgrades in the future.

Nevertheless, the "no distortion at the top" result present in the previous models continues to hold here. In equation (19), $\alpha\left(n_{1}\right)=0$ eliminates the first term of the right hand side. Rewriting equation (23) and taking the derivative with respect to $n$ gives us $\psi^{\prime}(n)=-\xi^{\prime}(n) \alpha(n) \phi(x(n))-\xi(n) \alpha^{\prime}(n) \phi(x(n))-\xi(n) \alpha(n) \phi^{\prime}(x(n)) x^{\prime}(n)$. Evaluating at $n=n_{1}$ and comparing to equation (22) evaluated at the same point proves that $\xi\left(n_{1}\right)=0$. This eliminates the second term on the right hand side of equation (19), showing that the quality is set such that the marginal cost equals the valuation for the top type, without any distortion.

We now prove an intriguing result: the firm is better off if the consumer becomes forward looking instead of myopic. In reality, we see firms recognizing this effect: firms selling running shoes have web sites with information about running, where one can keep a running diary and have access to other training aids. Car makers encourage test drives of their luxury models and hype their features. When the consumer can realize the gains of upgrading, the firm has less need to push higher quality onto low types at low prices and can make a larger profit.⿴囗十

\section{Proposition 5 Total profits are larger when consumers are forward looking.}

\section{Proof}

The solution when the consumer is myopic (and the firm forward looking) we denoted by $\left(x^{M}(),. p^{M}().\right)$. Let $\left(x^{F}(),. p^{F}().\right)$ denote the solution with forward looking consumers (and forward looking firms). We are going to prove that profits from the $F$ case are larger than profits from the $M$ case. We will do this by fixing the solution to the $M$ case $\left(x^{M}(),. p^{M}().\right)$

\footnotetext{
${ }^{11}$ This might suggest that consumers have the incentive to pretend to be myopic. In a previous version of the paper, we discuss an example which shows that there is a tradeoff for consumers (i.e. their incentives are not unambiguous). Two effects are present. Since the lowest type has larger rents (to be extracted) when forward looking, it is advantageous to pretend to be myopic. On the other hand, the interests of the monopolist and the forward looking agent may be aligned more than that of the monopolist and the myopic agent in the sense that both prefer upgrades. Hence, pretending to be myopic may lead to less upgrades and a dissappearance of surplus (for both the monopolist and the agent).
} 
and showing that a schedule with slightly higher prices $\left(x^{M}(),. p^{M}()+.\varepsilon\right)$ satisfies the constraints of the $F$ case.

From the value function for a forward looking customer:

$$
(\rho+\delta) V\left(n_{0}\right)=\underbrace{n_{0} x^{M}\left(n_{0}\right)-p^{M}\left(n_{0}\right)}_{=0}+\alpha\left(n_{0}\right) \phi\left(x^{M}\left(n_{0}\right)\right) V^{\prime}\left(n_{0}\right)+\delta \int_{n_{0}}^{n_{1}} V(m) g(m) d m
$$

It therefore follows that $V\left(n_{0}\right)>0$ and there exists an $\varepsilon>0$ such that the pricing schedule $p^{M}+\varepsilon$ still satisfies the IR constraint.

Next, consider the IC constraint. We first argue that a type $n$ who is offered the menu $\left(x^{M}(n), p^{M}(n)+\varepsilon\right)$ will not choose $\left(x^{M}\left(n^{\prime}\right), p^{M}\left(n^{\prime}\right)+\varepsilon\right)$ for some $n^{\prime}<n$. It is straightforward to see that

$$
n x^{M}(n)-p^{M}(n) \geq n x^{M}\left(n^{\prime}\right)-p^{M}\left(n^{\prime}\right) \quad \forall n^{\prime}<n
$$

implies that (using the fact that $d x^{M}(n) / d n \geq 0$ ):

$$
\begin{gathered}
n x^{M}(n)-p^{M}(n)-\varepsilon+\alpha(n) \phi(x(n)) V^{\prime}(n)+\delta \int_{n_{0}}^{n_{1}} V(m) g(m) d m \geq \\
n x^{M}\left(n^{\prime}\right)-p^{M}\left(n^{\prime}\right)-\varepsilon+\alpha(n) \phi\left(x\left(n^{\prime}\right)\right) V^{\prime}(n)+\delta \int_{n_{0}}^{n_{1}} V(m) g(m) d m \quad \forall n^{\prime}<n
\end{gathered}
$$

Secondly, in the appendix we prove that the firm will only profit from a customer overstating his type, i.e. if a type $n$ chooses $\left(x^{M}\left(n^{\prime}\right), p^{M}\left(n^{\prime}\right)+\varepsilon\right)$ rather than $\left(x^{M}(n), p^{M}(n)+\varepsilon\right)$, where $n<n^{\prime}$ the firm makes larger profits. Hence the profits in the $F$ case are at least $\varepsilon>0$ higher than in the $M$ case.

Q.E.D.

\section{Conclusion}

This paper develops a framework for analyzing dynamic adverse selection problems where types may change over time depending on the schedule the principal offers. The key element used in order to attain tractability is that agents are anonymous, which is often the case. We apply the model to monopoly pricing, analyzing price discrimination for experience and addictive goods. We find that the monopolist will increase quality for low types to encourage type upgrading. It may offer substantial discounts at a loss in order to reinforce this effect. For forward looking consumers, the adverse selection effect distorts quality upwards in contrast to the traditional static (and myopic) case. Moreover, the monopolist has incentives to make consumers forward looking, so that it need not sacrifice rents to encourage upgrading.

The methodology we develop here could be useful for other dynamic mechanism design problems where learning by doing plays an important role. A natural extension of the model would look at price discrimination when addictions are harmful. In addition, one might look at the regulation of a firm where marginal costs decrease with know-how and previous production or repeated auctions where consumers learn their demand for the good (as in electricity sales). 


\section{Appendix}

\subsection{Characterization of the Forward Looking solution}

We first prove a corollary to proposition 3:

Corollary 1 For functions $V($.$) that are twice differentiable we find that there exists c>0$ such that $V^{\prime}(n) \geq c$.

Proof: Consider the differential equation (which follows from (18))

$$
V^{\prime \prime}(n)-\beta(n) V^{\prime}(n)=-\gamma(n)
$$

where

$$
\begin{aligned}
\beta(n) & =\frac{\rho+\delta-\alpha^{\prime}(n) \phi(x(n))}{\alpha(n) \phi(x(n))}>0 \\
\gamma(n) & =\frac{x(n)}{\alpha(n) \phi(x(n))}>0
\end{aligned}
$$

The solution can then be written as

$$
V^{\prime}(n)=\left(C-\int_{n_{0}}^{n} \gamma(t) e^{-\int_{n_{0}}^{t} \beta(s) d s} d t\right) e^{\int_{n_{0}}^{n} \beta(t) d t}
$$

Next note that $\alpha\left(n_{1}\right)=0$ implies that $V^{\prime}\left(n_{1}\right)=\frac{x\left(n_{1}\right)}{\rho+\delta-\alpha^{\prime}\left(n_{1}\right) \phi\left(x\left(n_{1}\right)\right)}>0$. Because $\gamma(n)>0$, if $V^{\prime}(n)$ would become non-positive, it would happen at $n_{1}$, but it does not. As $V^{\prime}(n)$ is strictly positive for $n_{1}$, it is strictly positive for all $n$. The other terms are all nonzero and finite and hence we find $V^{\prime}(n) \geq c$ for some $c>0$. (Note, however, that it does not follow that $V^{\prime}(n)$ is decreasing in $n$ ).

Now we prove that the solution must have the constraint $V^{\prime}(n) \geq c$ binding only on an interval that begins at $n_{0}$ (or may only bind at $n_{0}$ ). $\mathrm{F}$ This allows us to conclude that $\psi(n) \geq 0$ for all $n$.

I. There is no solution where $\psi\left(n_{0}\right)=\psi\left(n_{1}\right)=0$ and $V^{\prime}(n)>c$ for all $n$

Proof by contradiction: assume $\psi\left(n_{0}\right)=\psi\left(n_{1}\right)=0$ and $V^{\prime}(n)>c$ for all $n$. Then:

$$
\psi(n)=\left(K+\int_{n}^{n_{1}} \rho(1-F(s)) e^{\int_{n_{0}}^{s} \frac{\rho+\delta-\alpha^{\prime} \phi(x)}{\alpha \phi(x)} d u} d s\right) e^{-\int_{n_{0}}^{n} \frac{\rho+\delta-\alpha^{\prime} \phi(x)}{\alpha \phi(x)} d s}
$$

where $K$ is the constant from integration. From $\psi\left(n_{1}\right)=0$, we have $K=0$ and $\psi(n) \geq 0$ for all $n$.

\footnotetext{
${ }^{12}$ This proof uses the necessary conditions that (i) $\psi\left(n_{0}\right)=0$ if $V^{\prime}\left(n_{0}\right)>c$, and $\psi\left(n_{0}\right) \geq 0$ if $V^{\prime}\left(n_{0}\right)=c$ and (ii) $\psi\left(n_{1}\right)=0$ if $V^{\prime}\left(n_{1}\right)>c$, and $\psi\left(n_{1}\right) \leq 0$ if $V^{\prime}\left(n_{0}\right)=c$. A derivation of these conditions can be found in Kamien and Schwartz (1991).
} 
From $\psi\left(n_{0}\right)=0$, we have $\int_{n_{0}}^{n_{1}} \rho(1-F(s)) e^{\int_{n_{0}}^{s} \frac{\rho+\delta-\alpha^{\prime} \phi(x)}{\alpha \phi(x)} d u} d s=0$. Since the integral must be positive, this is a contradiction.

II. It can't be the case that $\psi\left(n_{1}\right)<0$ and $V^{\prime}\left(n_{1}\right)=c$.

Proof by contradiction: suppose $\psi\left(n_{1}\right)<0$. Using equation (23) and the fact that $\alpha\left(n_{1}\right)=$ $0, \xi\left(n_{1}\right) \rightarrow \infty$. But if $\xi\left(n_{1}\right) \rightarrow \infty$, then equation (19) indicates that $x\left(n_{1}\right) \rightarrow-\infty$, which cannot be optimal.

III. It can't be the case that $V^{\prime}(n)=c$ for an interior interval $\left[n_{a}, n_{b}\right]$ where $n_{a}>n_{0}$.

If this was the case, $V^{\prime}\left(n_{a}\right)=c$ and it must be that $V^{\prime \prime}\left(n_{a}-\varepsilon\right)<0$ where $\varepsilon$ is small. From the incentive compatibility equation (18), however, $V^{\prime \prime}\left(n_{a}-\varepsilon\right)<0$ implies $V^{\prime}\left(n_{a}-\varepsilon\right)<c$, which is a contradiction.

IV. The only case remaining is where $\psi\left(n_{0}\right)>0$ and $V^{\prime}\left(n_{0}\right)=c$

\subsection{Supplementary proof for proposition 5}

Here we will prove that a firm prefers customers to overstate their types. This completes the proof that profits from selling to myopic consumers are lower than profits from selling to forward looking consumers.

Proposition $6 \pi^{\prime}(n) \geq 0$.

Proof Suppose it is not true, i.e. there exists $\tilde{n}$ such that $\pi^{\prime}(\tilde{n})<0$. Then one of two cases must occur:

either $(\mathrm{I}) \pi^{\prime}(n) \leq 0$ for all $n \in\left[\tilde{n}, n_{1}\right]$

or (II) there exists $n^{\prime} \in\left\langle\tilde{n}, n_{1}\right]$ such that $\pi^{\prime}\left(n^{\prime}\right)>0$.

We argue that neither of these two cases can happen.

First, consider case (I). In this case, the profits of the firm are higher if all types $n \geq \tilde{n}$ are offered $p(\tilde{n}), x(\tilde{n})$ since in that case profits are constant per type (for $n \geq \tilde{n}$ ) and hence $\pi^{\prime}(n)=0$ for all $n \in\left[\tilde{n}, n_{1}\right]$ which implies higher profits than the original menu $(p(n), x(n))$ which featured $\pi^{\prime}(n)<0$.

Second, consider case (II). Since $\pi^{\prime}(\tilde{n})<0<\pi^{\prime}\left(n^{\prime}\right)$ there exist values for $n$ such that $\pi^{\prime}(n) \leq 0$ and $\pi^{\prime \prime}(n)>0$. Consider two values $n^{*}<n^{* *}$ which are close together and satisfy $\pi^{\prime}\left(n^{*}\right)<0=\pi^{\prime}\left(n^{* *}\right)$. As the two points are close together, one option for the firm is to offer $p\left(n^{*}\right), x\left(n^{*}\right)$ to type $n^{* *}$ as well, but presumably the firm can do better. Thus we have

$$
\begin{gathered}
(\rho+\delta) \pi\left(n^{* *}\right) \geq p\left(n^{*}\right)-c\left(x\left(n^{*}\right)\right)+\delta \int_{n_{0}}^{n_{1}} \pi(m) g(m) d m> \\
p\left(n^{*}\right)-c\left(x\left(n^{*}\right)\right)+\phi\left(x\left(n^{*}\right)\right) \alpha\left(n^{*}\right) \pi^{\prime}\left(n^{*}\right)+\delta \int_{n_{0}}^{n_{1}} \pi(m) g(m) d m=(\rho+\delta) \pi\left(n^{*}\right)
\end{gathered}
$$

However, this contradicts $\pi^{\prime}\left(n^{*}\right)<0$ as $\pi\left(n^{* *}\right)>\pi\left(n^{*}\right)$.

Thus it must be the case that $\pi^{\prime}(n) \geq 0$. 


\section{References}

Atkeson, A. and Lucas, R., "Efficiency and Inequality in a Simple Model of Efficient Unemployment Insurance," Journal of Economic Theory 66 (1995), 64-88.

Baron, D, and Besanko, D., "Regulation and Information in a Continuing Relationship," Information Economics and Policy 1 (1984), 267-302.

Becker, G. S. and Murphy, K. M., "A Theory of Rational Addiction," Journal of Political Economy 96 (1988), 675-700.

Becker, G.S., Grossman, M., and Murphy, K.M., "An Empirical Analysis of Cigarette Addiction," NBER Working Paper \#3322 (1994).

Deneckere, R. J. and McAfee, R.P., "Damaged Goods," Journal of Economics and Management Strategy 5 (1996), 149-174.

Driskill, R. and McCafferty, S., "Monopoly and Oligopoly Provision of Addictive Goods," International Economic Review 42 (2001), pp. 43-72

Fethke, G. and Jagannathan, R., "Habit Persistence, Heterogeneous Tastes, and Imperfect Competition," Journal of Economic Dynamics Control 20 (1996), 1193-1207.

Freixas, X., Guesnerie, R. and Tirole, J., "Planning under Incomplete Information and the Ratchet Effect," Review of Economic Studies 52 (1985), 173-192.

Fudenberg, D. and Tirole, J. Game Theory (MIT Press, 1991)

Gabszewicz, J., Pepall, L., and Thisse, J-F. "Sequential Entry with Brand Loyalty caused by Consumer Learning-by-Using," Journal of Industrial Economics 40 (1992), 397-416.

Green E. "Lending and the Smoothing of Uninsurable Income," in Prescott, E. and Wallace, N. (editors) Contractual Arrangements for International Trade. University of Minnesota Press, 1987 .

Kamien, M.I., and Schwartz, N.L. Dynamic Optimization. (Amsterdam: North Holland, 1991).

Keller, K.L. Strategic Brand Management (New Jersey: Pearson Education, 2003).

Kocherlakota, Narayana, "Advances in Dynamic Optimal Taxation," University of Minnesota mimeo 2005.

Laffont, J.J., and Tirole, J., "The Dynamics of Incentive Contracts," Econometrica 56 (1988), 1153-1175.

Lewis, T. R., and Yildirim, H., "Learning by doing and dynamic regulation," RAND Journal of Economics 33 (2002), 22-36

Schlee, E. "Buyer experimentation and introductory pricing," Journal of Economic Behavior $\&$ Organization 44 (2001), 347-362

Shapiro, C. "Optimal Pricing of Experience Goods," Bell Journal of Economics 14 (1983), 497-507. 
Shapiro, C.and Varian, H. Information Rules: A Strategic Guide to the Network Economy (Cambridge: Harvard Business School Press, 1998).

Showalter, M., "Firm Behavior in a Market with Addiction: The Case of Cigarettes," Journal of Health Economics 18 (1999), 409-427.

Skreta, V., "Sequentially Optimal Mechanisms," UCLA mimeo, 2005.

Thomas, J. and Worrall, T., "Income Fluctuations and Asymmetric Information: An Example of a Repeated Principal-Agent Problem," Journal of Economic Theory 51 (1990), 367390.

Townsend, R.M. "Optimal Multiperiod Contracts and the Gain from Enduring Relationships under Private Information," Journal of Political Economy 90 (1982), 1166-1186. 